\title{
Quantitative Measuring Analysis Method and Mechanism of Wear Particle Settlement
}

\author{
Kaituo Zhang ${ }^{1}$, Zhiyong Lv ${ }^{2}$ \\ ${ }^{1}$ School of Automation, Wuhan University of Technology, Wuhan, Hubei, 430070, China \\ ${ }^{2}$ School of Energy and Power Engineering, Wuhan University of Technology, Wuhan, Hubei, 430070, China
}

\begin{abstract}
The size and distribution of wear particle in lubricating oil, as important numerical information available in ferrography, is one of the key indexes in wear diagnosis. In this paper, a new method for measuring the size and distribution of abrasive particles is proposed. First, all the abrasive fluid is left standing until all the abrasive particles are precipitated to the bottom. Then, the measuring container is inverted and the whole precipitation process of abrasive particles is recorded by magnetic induction instrument. And according to the precipitation analysis of the wear particle, the following results were obtained:1) At the initial stage of the particle settlement, the gravity, the buoyancy and the drag force of the oil achieve balance quickly, the time and distance of the wear particle moving at a constant velocity can be neglected. 2) The settling velocity is related to the diameter and specific gravity of the wear particle as well as the specific gravity and viscosity of the oil, the distribution of the wear particle is proportional to the square of the diameter of the particle, using the magnetic induction technology, the distribution of particle can be measured by settling time for different sizes of wear particles. 3) Measure the wear particle oil directly, there are different sizes of particles settlement in the bottom at the same time, which causes the difficulty in identifying the size of the particle settlement. The particle should be settled first, and then inverted, settling the particle in accordance with the order from large to small, which facilitates the measurement of different sizes of the particles, different times correspond to different sizes of the particles. 4) The bigger the particle is, the more accurate the measurement and counting is, the smaller the particle is, the bigger the error is.
\end{abstract}

\section{Introduction}

The size and distribution of wear particle in lubricating oil, as important numerical information available in ferrography, is one of the key indexes in wear diagnosis. The study on the wear particle concentration in the whole life cycle of the machine means the distribution of wear particle concentration over a continuous period, reflects the relationship between wear particle concentration and time. Torben Kjer considered the relationship between wear rate and concentration of large and small particles separately as well as the variation in wear rate during the running-in period[1]. Wojciech Szymczyk presented the methods and the related general mathematical model of concentration variation rates of particles in lubricating during the life of a mechanism[2]. C.S. Pande gets the grain-size distribution by both obtaining a numerical solution of Fokker-Planck equation in a stochastic model and performing computer simulations[3]. X.J. Lu describes the dynamic process of wear particle concentration in lube oil cycle system by transfer function[4].

At present, the measurement of wear particle coverage area usually uses ferrograph. The traditional ferrograph, such as Analytical Ferrograph (AF), Direct Reading Ferrograph (DRF), Online Direct Reading Ferrograph (ODRF) and so on, work efficiently in measuring the coverage area, more exactly, the coverage area of small particles AS and the coverage area of large particles AL. The basic principle of DRF is that, as oil entering the glass sediment tube slowly, under the effect of particles' own gravity, the permanent magnetic force and viscosity resistance, the wear particles precipitate on the inner wall of tube according to its own size[5].

\section{Theoretical Analysis}

\subsection{Theoretical Analysis of Uniform Settlement}

First, we analyze the second stage, for small particles, this settling process can be considered as uniform settling, where the resultant force is zero, and $\mathrm{a}=0$. The wear particles are balanced by gravity, buoyancy and drag force [6], using $u_{t}$ represent the particle velocity at this time: the wear particle settling process is divided into two stages: 1) accelerate settling process 2) uniform settling process.

\footnotetext{
"Corresponding author's e-mail: 2644401716@qq.com
} 


\subsection{Force Analysis of Settling Process}

The first stage: In the case of variable acceleration motion, as the drag force increased with the speed, until the gravity, the buoyancy and the drag force achieved balance. The force of the wear particle is shown in Fig. 1.

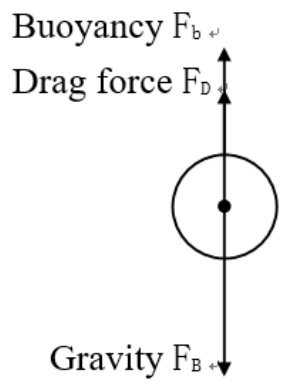

Fig. 1. Force analysis of wear particle in the oil.

The gravity:

$$
\mathrm{F}_{\mathrm{B}}=\mathrm{mg}=\frac{\pi}{6} \rho_{\mathrm{p}} d_{p}^{3}
$$

The buoyancy:

$$
\mathrm{F}_{\mathrm{B}}=\mathrm{mg}=\frac{\pi}{6} \rho d_{p}^{3}
$$

Where $\rho, \rho_{p}$ is the density of particle and liquid. The drag force of the oil to the particle $F_{D}$ :

$$
F_{D}=\xi_{D} \frac{\rho u^{2}}{2} A
$$

According to Newton's second law:

$$
F_{B}-F_{b}-F_{D}=m a
$$

Where $\xi_{D}$ is the drag coefficient, $A$ is the projected area of the particle in the flow direction, and $a$ is the acceleration of the wear particle.

$$
\frac{\rho_{p} d_{p}^{3}}{6}-\rho \frac{d_{p}^{3}}{6} g-\xi_{D} \frac{\rho_{p} u_{t}^{2}}{2} \frac{\pi}{4} d_{p}^{2}=0
$$

The formula for calculating the speed is obtained:

$$
u_{t}=\sqrt{\frac{4 d_{p}\left(\rho_{p}-\rho\right) g}{3 \rho \xi_{D}}}
$$

Where $d_{p}$ is the diameter of the particles.

According to fluid mechanics: $\operatorname{Re}_{p} \leq 2$, laminar flow area, also known as Stokes area, $\xi_{D}$ is:

$$
\xi_{\mathrm{D}}=\frac{24}{\mathrm{Re}_{p}}
$$

Where the drag coefficient $\xi_{D}$ is a function of the Reynolds number $\operatorname{Re}_{p}=d_{p} u_{t} \rho / \mu, \mu$ is the dynamic viscosity $(\mathrm{Pa} \cdot \mathrm{s})$. We can get:

$$
u_{t}=\frac{d_{p}^{2}\left(\rho_{p}-\rho\right) g}{18 \mu}
$$

In the process of settlement, the different particle settlement is mutual interference, where $d_{p}$, there are granular, flaky and other irregular shape, the calculation should be substituted into the equivalent diameter, and the following formula to amend:

$$
u_{T}^{\prime}=u_{T} \frac{1}{1+\alpha i^{\frac{1}{3}}}
$$

Where $i$ is volume ratio of wear particle to liquid, $\alpha$ is shape influence factor and a function of $i$, the relationship is shown in Table 1.

Table 1. Shape influence factor of particle [8].

\begin{tabular}{|c|c|c|}
\hline $\begin{array}{c}\text { Particle } \\
\text { conditions }\end{array}$ & $i$ & Value $\alpha$ \\
\hline Irregular particle & $0.15 \sim 0.5$ & $\alpha=1+305 i^{2.84}$ \\
\hline Spherical particle & $0.2 \sim 0.5$ & $\alpha=1+229 i^{3.43}$ \\
\hline $\begin{array}{c}\text { Very thin } \\
\text { suspension }\end{array}$ & $i<0.15$ & $\alpha=1 \sim 2$ \\
\hline
\end{tabular}

According to the above reasoning, under the condition of $20^{\circ} \mathrm{C}$, for iron wear particle (proportion is $8.9 \mathrm{~kg} / \mathrm{m}^{3}$ ),

\begin{tabular}{|c|c|c|c|c|c|c|c|c|c|c|c|c|}
\hline $\begin{array}{l}\text { Equivalent } \\
\text { Diameter }\end{array}$ & 2 & 5 & 10 & 15 & 20 & 25 & 3 & 4 & 5 & 75 & 100 & 1000 \\
\hline $\mathrm{T}$ & 3.9E-09 & $2.4 \mathrm{E}-08$ & $9.8 \mathrm{E}-08$ & 2.2E-07 & $3.9 \mathrm{E}-07$ & $6.2 \mathrm{E}-07$ & $9.0 \mathrm{E}-07$ & $1.6 \mathrm{E}-06$ & 2.6E-06 & $5.6 \mathrm{E}-06$ & $9.9 \mathrm{E}-06$ & $9.9 \mathrm{E}-04$ \\
\hline S & 7.7E-21 & $3.0 \mathrm{E}-19$ & $4.8 \mathrm{E}-18$ & $2.4 \mathrm{E}-17$ & 7.7E-17 & $1.9 \mathrm{E}-16$ & $3.9 \mathrm{E}-16$ & $1.2 \mathrm{E}-15$ & $3.0 \mathrm{E}-15$ & $1.5 \mathrm{E}-14$ & $4.9 \mathrm{E}-14$ & $4.9 \mathrm{E}-10$ \\
\hline $\mathrm{u}$ & $1.7 \mathrm{E}-08$ & $1.0 \mathrm{E}-07$ & 4.3E-07 & $9.8 \mathrm{E}-07$ & $1.7 \mathrm{E}-06$ & $2.7 \mathrm{E}-06$ & $3.9 \mathrm{E}-06$ & 7.0E-06 & $1.1 \mathrm{E}-05$ & $2.5 \mathrm{E}-05$ & $4.4 \mathrm{E}-05$ & 4.4E-03 \\
\hline
\end{tabular}
and oil (proportion is $0.8 \mathrm{~kg} / \mathrm{m}^{3}$ ), dynamic viscosity 1 , analyze the settling time and distance of wear particle with equivalent diameter of $2,5,10,15,30,25,30,40,50,75$, 100 and $1000 \mu \mathrm{m}$.

As can be seen from the Table 2, the time, distance and velocity are particularly small, so from the beginning to the force balance, the time and distance of the wear particle for uniform motion can be neglected.

Table 2. Time, distance and velocity of particle settlement reached force balance.

\subsection{Quantitative Analysis Method of Wear Particle Settlement}

During the measurement, the settling time of the different wear particles can be measured and analyzed by using the formula ( 8 ). If precipitate the wear particle before inversion, according to the formula (8), the large particle drop quickly while the small particle drop slowly, when the container is inverted, all the particles are settled from 
the top, the large particle settle to the bottom faster than the small particle necessarily.

If the height of the container is $\mathrm{H}$, the settling velocity $\mathrm{u}$ of the particle is obtained according to formula (8), ignoring the start time of the wear particle, and the settling time is:

$$
t=\frac{18 \mu H}{d_{p}^{2}\left(\rho_{p}-\rho\right) g}
$$

Through the analysis, the specific settling time is shown in Table 3.

Table 3. Settling time analysis of different wear particle for different height containers.

\begin{tabular}{|c|c|c|c|}
\hline $\begin{array}{c}\text { Time }(\mu \mathrm{m}) \\
\text { Diameter }\end{array}$ & T100(min) & T50(min) \\
\hline 2 & 95424 & 47712 & 9542 \\
\hline 5 & 15267 & 7633 & 1526 \\
\hline 10 & 3816 & 1908 & 381 \\
\hline 15 & 1696 & 848 & 95 \\
\hline 20 & 954 & 477 & 61 \\
\hline 25 & 610 & 305 & 42 \\
\hline 30 & 424 & 212 & 169 \\
\hline 40 & 238 & 119 & 6 \\
\hline 50 & 152 & 76 & 3 \\
\hline 75 & 67 & 33 & 0.038 \\
\hline 100 & 68 & 19 & \\
\hline 1000 & 0.38 & & 9.19 \\
\hline
\end{tabular}

From the analysis data in Table 3 , it can be seen that

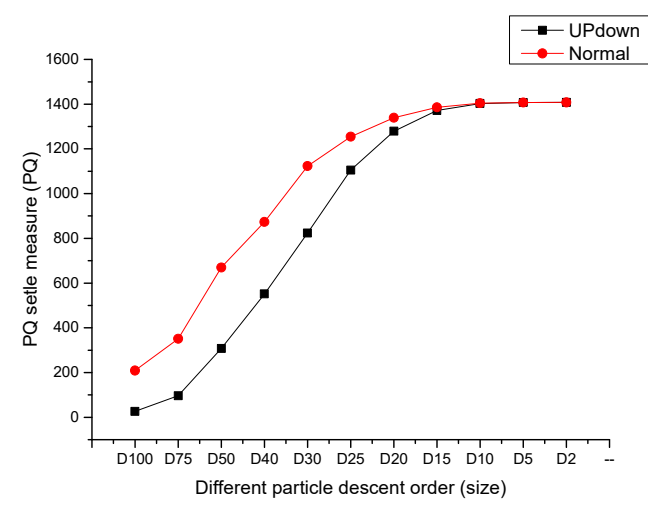

(a)
$50 \mathrm{~mm}$ is suitable height of the measuring device.

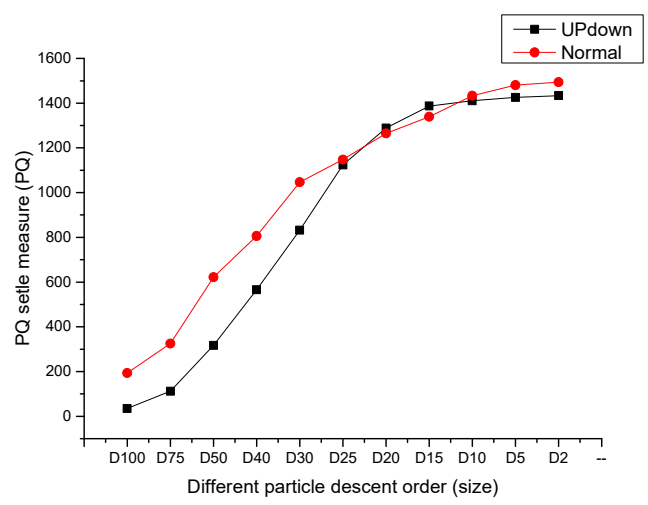

(b)

Fig. 2. PQ value comparison experiment of normal and inverted wear particle.

Fig.2(a) is the theoretical PQ value measured by the CSI device, Fig. 2(b) is the PQ value measured by the PQP measuring device, due to part of the particles hang into the container during the PQP measurement, and the instrument measurement error, Fig. 4(b) can not repeat the ideal curve of Fig. 4(a), but it still reflects the settling sequence and time characteristics of the particles.

\section{PQ Quantitative Measurement and Calibration}

Then according to the exponential curve of Time-PQ, and combine the output voltage changes caused by the different size of single wear particle in the pre-experiment, the PQ index can be obtained. The approximate number of different sizes of the particles can be obtained by analyze the settling time.

Aiming at the ferromagnetic characteristic of metal wear particles, at present, there are magnetic-type particles online monitoring PQ device, it is also a relatively common method of measuring particle. According to the above measuring method, the weights of different wear particles are measured, and compared with the PQ index, as shown in Fig.3, there is a good linear characteristic in the range of $0-0.03 \mathrm{~g}$. 


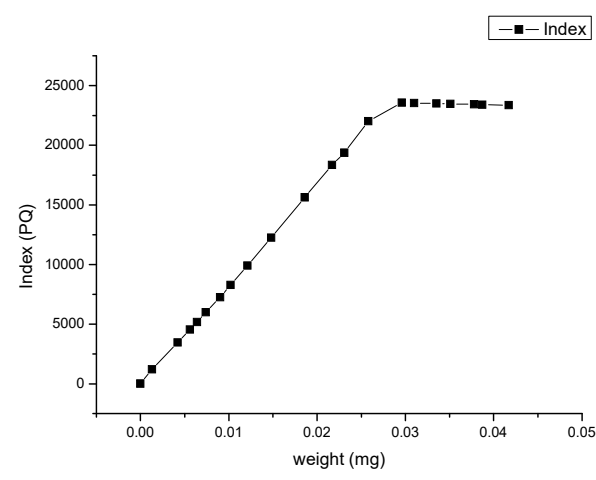

Fig. 3. Relationship between wear particle weight and PQ index.

According to the relationship between the equivalent diameter and the weight:

$$
\begin{gathered}
P Q=5.5+817638 * W \\
W=\frac{1}{6} \pi D^{3} \rho_{p}
\end{gathered}
$$

The fitting relationship between the individual particle weight and the wear particle PQ index is:

$$
P Q=5.5+136273 \pi D^{3} \rho_{p}
$$

It can be seen from Fig.3, when the weight of the oil particle more than $0.025 \mathrm{mg}$, the curve is not a linear relationship, when the weight of the oil particle more than $0.03 \mathrm{mg}$, the measurement is saturated. When the overflow situation occurs, we can dilute the concentration of particle to prevent the overflow, the specific number of dilution as the case and experience.

\section{Analysis of $P Q$ Inversion Settlement Method}

\subsection{Differential Detection Method of Different Sizes of Wear Particles}

If the diameters of wear particles are 2, 5, 10, 15, 30, 25, $30,40,50,75,100$ and $1000 \mu \mathrm{m}$, their weights are expressed as w1, w2, w3, w4, w5, w6, w7, w8, w9, w10, $\mathrm{w} 11$, and $\mathrm{w} 12$, and the settling time is $\mathrm{t} 1, \mathrm{t} 2, \mathrm{t} 3, \mathrm{t} 4, \mathrm{t} 5$, $\mathrm{t} 6$, $\mathrm{t} 7, \mathrm{t} 8, \mathrm{t} 9, \mathrm{t} 10, \mathrm{t} 11, \mathrm{t} 12$.

The index PQ1, PQ2...PQ12 measured at different time correspond to Q1, Q2...Q12, the correspondence relationship can be obtained according to the formula (3$3)$.

$$
Q_{n}=\sqrt[3]{\frac{P Q_{n}-5.5}{136273 \pi \rho_{p}}} \quad(\mathrm{n}=1,2,3 \ldots 12)
$$

The total weights of the different wear particle are:

$$
\left\{\begin{array}{l}
W 1=Q 12-Q 11 \\
W 2=Q 11-Q 10 \\
\cdots \\
W 11=Q 2-Q 1 \\
W 12=Q 1
\end{array}\right.
$$

According to the above analysis, we can get the mapping relation between different diameter of the wear particle settlement and time.

\subsection{Weight calculation of individual particle}

The ratio of the length, width and height of the different wear particles is different, and the distribution of the wear particle is different at different times[9]. However, it is impossible to obtain the type distribution of wear particle when the results were not obtained. Generally speaking, most of the particles are wear particles, it can only approximate that: the length, width and height $=\mathrm{a}: \mathrm{b}: \mathrm{c}=$ 15: 10: 1 , if calculated according to the ellipsoid, the relationship between weight and diameter is:

$$
\omega_{n}=V \rho_{p}=\frac{4 \pi}{3} a b c \rho_{p}=\frac{2 \pi D^{3} \rho_{p}}{225}
$$

Contrast analysis can be obtained according to the ratio of length, width and height and particle size, it needs to multiply by a coefficient of $\mathrm{C}$, the coefficient is related to the distribution of length, width and height. The coefficient of this paper is obtained by CSI instrument comparison experiment, $\mathrm{C}$ is:

$$
C=\frac{P Q_{C S I}}{P Q_{n}}
$$

Where $P Q_{C S I}$ converted from CSI, $P Q_{n}$ measured by the PQP instrument.

\subsection{Comparative analysis of PQP measurement and CSI experiment}

In this experiment, a high $50 \mathrm{~mm}$ container was used for PQP measurement, The measurement time was measured according to the settling time of the different wear particle with height of $50 \mathrm{~mm}$ in Table 3 , and the corresponding wear particle PQ values were measured. As the total PQ contribution of the different wear particle is different, in order to compare the two different methods, we convert the results of the SIC measurements to PQ values, in this paper we can calculate the individual contribution of $P Q$ in the table according to formula (13) and the particle size, then sum the number of all particles and their contribution rate, and get the PQ value of a certain size of all particle converted by the CSI experiment.

$$
\triangle P Q_{C S I-n}=P Q_{i} \times N_{C S I-n}
$$

Where $\mathrm{n}$ is settling sequence, $P Q_{i} \quad$ is converted PQ values of individual wear particle.

According to the settling sequence, all the precipitated wear particles are summed according to time, and get the CSI theory inverted $P Q_{C S I-n}$ value of all settlement. The analysis process of the PQP instrument and the CSI instrument is exactly contrary, the measured PQ value is the sum of the large particle and small particle, if you want to get the PQ value of a certain kind of particle, the PQ value of each time need to subtract the last time PQ value in order to get the PQ value of the particle in this period. 


$$
\triangle P Q_{i}=P Q_{i}-P Q_{i-1}
$$

According to the formula (15), the number of particle $N_{n}$ is:

$$
N_{n}=\frac{\Delta P Q_{i}}{P Q_{i}}
$$

\section{The Influence Analysis of PQ Measurement Error on Particle Counting}

The accuracy of the measurement depends on the measuring progress of the $\mathrm{PQ}$ instrument, and the magnetic induction of the magnetic coil is very strong, we analyze the relationship between the error of PQ and the precision of measurement. When the equivalent diameter the wear particle is 5-100 um, the contribution rate of PQ index is different for different sizes of wear particles if $10 \%$ of the error is acceptable. In this experiment, the measurement accuracy of PQ equipment is not high, which leads to two aspects of the error.

\section{Conclusion}

1)This paper theoretically analyzed the balance process of the buoyancy, the gravity and the drag force of the wear particle in the oil. At the initial stage of the particle settlement, the gravity, the buoyancy and the drag force of the oil achieved balance quickly, the balance time can almost be ignored. Time, distance, velocity are particularly small, so from the beginning to the force balance, the time and distance of the wear particle moving at a constant velocity can be neglected.

2)Through the force analysis of the settlement, the settling velocity of the wear particle can be considered as: The settling velocity is related to the diameter and specific gravity of the wear particle as well as the specific gravity and viscosity of the oil. The change of the specific gravity of the wear particle and the specific gravity and viscosity of the oil are generally small under normal temperature and normal pressure, so the distribution of the wear particle measured by settlement is proportional to the square of the diameter of the wear particle, using the magnetic induction technology, the distribution of particle can be measured by settling time for different sizes of wear particles.

\section{References}

1. Toren Kjer.: Wear rate and concentration of wear particles in lubricating oil. Wear 67(2), 217-226 (1981).

2. Wociech Szymczyk.: Variations in wear particle concentration in lubricating oil. Wear 80(1), 121-124 (1982).

3. C.S. Pande. K.P. Cooper. G.B. McFadden.: Grain size distribution in two dimensions in the long time limit. Acta Materialia 56 (18), 5304-5311 (2008).

4. X.J. Lu. X. Wu. M.Q. Jing. Y.B. Xie.: Research on
Dynamic Process of Wear Particle Concentration in Oil. Lubrication Engineering. 15-17 (2003).

5. Yang Qiming.: Wear particle analysis: wear particle atlas and Ferrography. China Railway Publishing House. 101-105 (2002).

6. Xiong Guoli.: Another calculation method for sediment settling velocity. Journal of Chang'an University (Journal of Architecture and Civil Engineering). 71-74 (1986).

7. Wang Jian, Feng Chao, Ma Qiang, Wang Danlin, Wu Fengdou, Wang lan.: Study on suspend ability and suspension mechanism of inorganic particles plugging agents. Speciality Petrochemicals 33(1), 5558 (2016).

8. Sun Tichang.: Solid-liquid separation. Central South University Press. 251 (2011).

9. Lv Zhiyong.: Wear particle image digital detection method. Science Press. 146-149 (2010). 